\title{
Gravity cells as a necessary link in string theory. The formula for the gravitational constant, the formula for the mass of the electron, the formula for the minimum distance of the gravitational interaction. The coincidence of the results obtained by the formulas and the experimental data.
}

Andrey Chernov ( $\sim$ and8591@gmail.com )

none Company/Institution

Article

Keywords: Black hole, string theory, gravitational cells, gravitational constant formula, electron mass formula, electron mass calculation, elementary charge, Schwarzschild radius formula, gravitational cell mass, standing gravitational wave, gravitational string, gravitational quantum, gravitational quantum energy formula, vibrational velocity formula gravitational strings, Casimir effect, minimum distance of gravitational interaction, minimum length of a standing gravitational wave,light speed formula

Posted Date: August 16th, 2021

DOI: https://doi.org/10.21203/rs.3.rs-622706/v11

License: (c) (i) This work is licensed under a Creative Commons Attribution 4.0 International License. Read Full License 
Gravity cells as a necessary link in string theory. The formula for the gravitational constant, the formula for the mass of the electron, the formula for the minimum distance of the gravitational interaction. The coincidence of the results obtained by the formulas and the experimental data.

\author{
Author Andrey Chernov \\ Address: Andrey Chernov, 16 Polenova str, apt.7, Irkutsk, 664007, Russia. \\ cell phone: +7 9645407 298. E mail: and8591@gmail.com
}

\title{
Content
}

1. Abstract -2 pg.

2. Introduction $-3-4 \mathrm{pg}$.

3. Methods $-4-17 \mathrm{pg}$.

4. Results and discussion $-17-19 \mathrm{pg}$.

5. Conclusions $-19-21 \mathrm{pg}$.

6. Declarations $-22 \mathrm{pg}$. 


\section{Abstract.}

In this study, a new concept is introduced into physics - gravitational cells.

The gravitational cell hypothesis was organically integrated into string theory. As a result, using the Schwarzschild radius formula and the Coulomb formula, a gravitational formula in the region of black holes was obtained on the basis of two fundamental constants, and its exact value was determined. The value of the "usual" gravitational constant was also confirmed and the mass of the gravitational cell was obtained.

The introduction of the hypothesis of gravitational cells into string theory made it possible to apply Planck's formula to gravitational interaction. As a result, a formula for the energy of a gravitational quantum and a formula for the vibrational velocity of a gravitational string were obtained. On this basis, the formula for the mass of the electron was obtained and its value was calculated, which coincided with the experimental mass of the electron. The exact minimum distance of the gravitational interaction was determined by the formula for the vibrational velocity of the gravitational string. This calculated minimum distance completely coincided with the known experimental data obtained when determining the Casimir effect (force).

Keywords. Black hole, string theory, gravitational cells, gravitational constant formula, electron mass formula, electron mass calculation, elementary charge, Schwarzschild radius formula, gravitational cell mass, standing 
gravitational wave, gravitational string, gravitational quantum, gravitational quantum energy formula, vibrational velocity formula gravitational strings, Casimir effect, minimum distance of gravitational interaction, minimum length of a standing gravitational wave.

\section{Introduction.}

The main goal of this study is to embed the hypothesis of gravitational cells in string theory and obtain, on this basis, specific results that can be compared with experimental data. Therefore, in the introductory part, it is better to highlight the issues of modern physics in the main areas of this article. Let's start with the gravitational constant.

In physics, there have been several attempts to propose scientifically substantiated formulas for calculating the gravitational constant. All the formulas presented by the authors are quite complex, and they necessarily contain additional (often artificial) coefficients and indicators, without which these formulas do not work.

String theory is a branch of theoretical physics that studies the dynamics of the interaction of objects not as point particles, but as one-dimensional extended objects, the so-called quantum strings. String theory combines the ideas of quantum mechanics and the theory of relativity. The theory that arose to describe hadronic physics, but did not quite fit for this, turned out to be a kind of experiment in a vacuum. The main problem of string theory is expressed in a huge 
variety of possible solutions, the so-called "landscape problem". This significant circumstance, according to such famous scientists as Lee Smolin and David Gross, takes string theory out of the framework of scientific theory. In this paper, I will try to prove that the introduction of gravitational cells into string theory can give concrete results.

\section{Methods.}

The study begins with the Coulomb formula. Let's write this formula for the case of interaction of two opposite elementary charges:

$$
f=k \cdot \frac{q_{0} \cdot q_{0}}{r^{2}} \quad \text { or } \quad f=k \cdot \frac{q_{0}^{2}}{r^{2}}
$$

Where $\boldsymbol{f}$ is the force of attraction of two elementary charges.

where $\boldsymbol{q}_{\mathbf{0}}$ is an elementary charge, $1,60217733 \cdot 10^{-19} \mathrm{C}$.

$\boldsymbol{k}$ is the coefficient of proportionality, $\boldsymbol{k}=\frac{1}{4 \pi \varepsilon_{0} \varepsilon}$. Where $\frac{1}{4 \pi \varepsilon_{0}}$ is a constant equal to $\mathbf{9} \cdot \mathbf{1 0}^{\mathbf{9}} \frac{\mathrm{kg} \mathrm{m}}{\mathrm{C}^{2} \mathrm{~s}^{2}}$, and $\boldsymbol{\varepsilon}$ is the relative dielectric constant of the medium.

$\boldsymbol{r}$ is the distance between charges, $\mathrm{m}$.

The value of the proportionality coefficient $\boldsymbol{k}$ depends on the medium.

Maximum value $\boldsymbol{k}=\mathbf{9} \cdot \mathbf{1 0}^{\mathbf{9}} \frac{\mathrm{kg} \mathrm{m}}{\mathrm{C}^{2} \mathrm{~s}^{2}}$ in vacuum (where $\boldsymbol{\varepsilon}=1$ ), for alcohol $\boldsymbol{k}=2,6 \cdot$ $10^{8}$ (where $\boldsymbol{\varepsilon}=35$ ), for water $\boldsymbol{k}=1,1 \cdot 10^{8}$ (where $\boldsymbol{\varepsilon}=80$ ), for copolymers $\boldsymbol{k} \geq$ $10^{4}$ (where $\boldsymbol{\varepsilon} \leq 105$ ), etc. Theoretically, $\boldsymbol{k}$ can approach its minimum value equal to 1 . But $\boldsymbol{k}<\mathbf{1}$ cannot be in any medium, because then the fractionality of elementary charges is allowed. Practical confirmation of this lies in the fact that 
science does not know substances where $\boldsymbol{\varepsilon}>\boldsymbol{9} \cdot \mathbf{1 0}^{\mathbf{9}}$. The maximum value of $\boldsymbol{\varepsilon}$ is recorded for metal nanoisland structures, $10^{7}-10^{8}$, where $\boldsymbol{k} \geq 90$.

Now let's move on to the gravitational interaction of such physical objects as black holes. (The result obtained here will then make it possible to pass to the gravitational interaction of ordinary bodies).

So, we have two black holes of mass $\boldsymbol{M}$ and $\boldsymbol{M}_{\mathbf{1}}$, located at a distance $\mathrm{r}$ from each other. The body of a black hole consists of many superdense cells with mass $\boldsymbol{m}_{\mathbf{0}}$ and charge $\boldsymbol{q}_{\boldsymbol{g}}$, numerically equal to the value of two elementary charges $\boldsymbol{q}_{\mathbf{0}}$, that is, $\boldsymbol{q}_{\boldsymbol{g}}=\mathbf{2} \boldsymbol{q}_{\mathbf{0}}$. Such a cell (let's call it a gravitational cell), in a black hole, is a tightly compressed pair of elementary particles: an electron and a proton. In this case, the mass of such a cell will be less than the total mass of a free proton and a free electron by $\Delta \boldsymbol{m}$ due to the release of energy during compression, where $\Delta \boldsymbol{m}=\boldsymbol{E} / \mathrm{c}^{2}$. The gravitational cells of one black hole $\boldsymbol{M}$ are connected by ultramicroscopic strings with the gravitational cells of another black hole $\boldsymbol{M}_{\mathbf{1}}$. As a result, a common gravitational field $\boldsymbol{E}$ is created in space, through which the interaction between black holes takes place along the gravitational strings. The field strength of the gravitational cell $\boldsymbol{g}_{\mathbf{0}}$ in the black hole region $\boldsymbol{M}$ is determined by the following formula:

$$
g_{0}=\frac{k_{0} q_{g 1} \cdot k_{0} q_{g 2}}{r^{2}} \quad \text { or } \quad g_{0}=k_{0}^{2} \frac{4 q_{0}^{2}}{r^{2}}
$$

where $\boldsymbol{g}_{\mathbf{0}}$ is the value of the gravitational field of one gravitational cell of a black hole, $\mathrm{m} / \mathrm{s}^{2}$. 
$\boldsymbol{k}_{\mathbf{0}}-$ proportionality coefficient, $\boldsymbol{k}_{\mathbf{0}}=\mathbf{1} \frac{\mathrm{m}^{3 / 2}}{\mathrm{C} \mathrm{s}}$,

$\boldsymbol{q}_{\mathbf{0}}$ - elementary charge $1,60217733 \cdot 10^{-19} \mathrm{C}$.

$\boldsymbol{q}_{\boldsymbol{g} \mathbf{1}}$ and $\boldsymbol{q}_{\boldsymbol{g} \mathbf{2}}$ are the charges of the gravitational cells of black holes, $\boldsymbol{q}_{\boldsymbol{g}}=\mathbf{2} \boldsymbol{q}_{\mathbf{0}}=$ $3,20435466 \cdot 10^{-19} \mathrm{C}$.

$\boldsymbol{r}$ - distance between cells, $\mathrm{m}$.

Thus, the gravitational field around the gravitational cell at a distance $\boldsymbol{r}=\mathbf{1}$

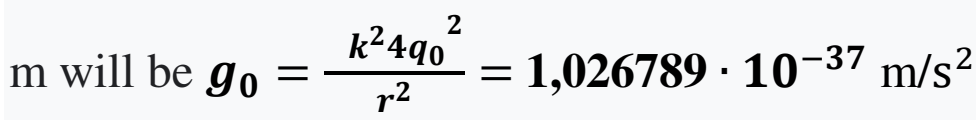

Formula (1-2) is similar to Formula (1-1). But if in the Coulomb formula the coefficient of proportionality in vacuum is $\boldsymbol{k}=\mathbf{9} \cdot \mathbf{1 0}^{\mathbf{9}}$, then in the formula (1-2) $\boldsymbol{k}_{\mathbf{0}}=\mathbf{1}$. The reason for such a large discrepancy in the coefficients is that the substance of the black hole is so strongly compressed that almost all lines of force of elementary charges are closed inside gravitational cells. And only an extremely small part of the lines of force go out from the cell, creating a gravitational field in the outer space. As a result of this circumstance, the coefficient of proportionality of elementary charges outside the gravitational cell decreases to its minimum threshold, that is, exactly to 1 . At the same time, the main electric field, with the coefficient of proportionality $\boldsymbol{k}=\mathbf{9} \cdot \mathbf{1 0}^{\mathbf{9}}$, remains closed between elementary charges inside the gravitational cells and therefore does not manifest itself in any way.

Now let's consider the interaction of two black holes $\boldsymbol{M}$ and $\boldsymbol{M}_{\mathbf{1}}$. The body of a black hole with mass $\boldsymbol{M}$ consists of a huge number of gravitational cells with 
mass $\boldsymbol{m}_{\mathbf{0}}$. Therefore, the total number of such cells will be: $\boldsymbol{n}=\frac{\boldsymbol{M}}{\boldsymbol{m}_{\mathbf{0}}}$. These gravitational cells form a common gravitational field in space, equal to $\boldsymbol{E}=\boldsymbol{g}_{\mathbf{0}}$. $\boldsymbol{n}$. Thus, the gravitational field $\boldsymbol{E}$ around a black hole $\boldsymbol{M}$ when it interacts at a distance $\mathrm{r}$ with another black hole will be:

$$
E=\frac{k_{0}^{2} 4 q_{0}^{2}}{r^{2}} \frac{M}{m_{0}} \quad \text { or } \quad E=\frac{k_{0}^{2} 4 q_{0}^{2}}{m_{0}} \frac{M}{r^{2}}
$$

The expression $\frac{\boldsymbol{k}_{\mathbf{0}}^{2} \mathbf{4 \boldsymbol { q } _ { 0 }}}{\boldsymbol{m}_{\mathbf{0}}}$ is the value of the gravitational constant $\boldsymbol{G}_{\mathbf{0}}$ for the case of interaction of two black holes. (Further, for better perception, the coefficient $\boldsymbol{k}_{\mathbf{0}}$ $=\mathbf{1}$ will not be displayed in the formulas). As a result, for $\boldsymbol{k}_{\mathbf{0}}=\mathbf{1}$, formula (1-4) will take the following form:

$$
E=G_{0} \frac{M}{r^{2}}, \text { where } G_{0}=\frac{4 q_{0}^{2}}{m_{0}}
$$

It follows that $\boldsymbol{m}_{\mathbf{0}}=\frac{\mathbf{4 q}_{\mathbf{0}}{ }^{2}}{\boldsymbol{G}_{\mathbf{0}}}$. It is possible to determine the value $\boldsymbol{m}_{\mathbf{0}}$ if we admit the possibility of equality $\boldsymbol{G}_{\mathbf{0}}=\boldsymbol{G}=\mathbf{6 , 6 7 4 3} \cdot \mathbf{1 0}^{-\mathbf{1 1}}$. In this case, we get $m_{0}=\frac{4 q_{0}{ }^{2}}{6,6743 \cdot 10^{-11}}=1,53842 \cdot 10^{-27} \mathrm{~kg}$. But such a calculation of the value $m_{0}$ cannot be considered correct, because the value of the gravitational constant $G_{0}$ in the region of the black hole may have a different value, that is, $\boldsymbol{G}_{\mathbf{0}} \neq \boldsymbol{G}$. Therefore, in order to correctly determine the value of $\boldsymbol{m}_{\mathbf{0}}$ and also the value of $\boldsymbol{G}_{\mathbf{0}}$, it is necessary to turn to the formula for the Schwarzschild gravitational radius:

$$
R=\frac{2 G_{0}}{c^{2}} \cdot M
$$


where $\boldsymbol{R}$ is the gravitational radius of a black hole, $\boldsymbol{G}_{\mathbf{0}}$ is the gravitational constant in the field of a black hole, $\boldsymbol{M}$ is the mass of a black hole, and $\boldsymbol{c}$ is the speed of light.

In this formula, of particular interest is the expression $\frac{2 G_{0}}{c^{\mathbf{2}}}$, which is measured in "m / kg", and denotes the specific length of the gravitational radius of a black hole. (In what follows, we denote $\frac{2 G_{0}}{c^{2}}$ as $\gamma$ ). And now a very important point. In space compressed to a point around a black hole, such a category as space does not exist. Therefore, in the Schwarzschild radius, all physical quantities merge into one quantity - mass, where the initial dimensional unit is the mass of the gravitational cell $\boldsymbol{m}_{\mathbf{0}}$. As a result of this circumstance, in the gravitational radius of the black hole, the numerical equality must be observed: $\boldsymbol{\gamma}=\boldsymbol{m}_{\mathbf{0}}$. Taking into account that $\boldsymbol{\gamma}=\frac{2 \boldsymbol{G}_{\mathbf{0}}}{\boldsymbol{c}^{\mathbf{2}}}$, and $\boldsymbol{m}_{\mathbf{0}}=\frac{\mathbf{4 q _ { 0 }}{ }^{2}}{\boldsymbol{G}_{\mathbf{0}}}$, we get the following equation: $\frac{2 G_{0}}{c^{2}}=\frac{4 q_{0}^{2}}{G_{0}}$. Let's solve it and as a result we get:

$$
\begin{aligned}
& G_{0}=\sqrt{2} q_{0} c=6,7927 \cdot 10^{-11} \frac{\mathrm{m}^{3}}{\mathrm{~s}^{2} \mathrm{~kg}} \\
& m_{0}=\frac{\sqrt{8} q_{0}}{c}=1,511593 \cdot 10^{-27} \mathrm{~kg}
\end{aligned}
$$

As you can see, $\boldsymbol{G}_{\mathbf{0}}$ differs from $\boldsymbol{G}=\mathbf{6}, \mathbf{6 7 4 3} \cdot \mathbf{1 0}^{\mathbf{- 1 1}}$ by $1,7 \%$. In this case, the value of $\boldsymbol{G}_{\mathbf{0}}$ is related to the mass of the gravitational cell $\boldsymbol{m}_{\mathbf{0}}$ by the following beautiful formula: $G_{0}=\frac{m_{0} c^{2}}{2}(1-9)$. 
Now consider the gravitational interaction of an ordinary (that is, not superdense) body $\boldsymbol{M}$ with another body $\boldsymbol{M}_{\mathbf{1}}$. The formula of the gravitational field around a body of mass $\boldsymbol{M}$ in this case will look like this:

$$
E=\frac{k^{2} 4 q_{0}^{2}}{r^{2}} \frac{M}{m}=\frac{k^{2} 4 q_{0}^{2}}{m} \frac{M}{r^{2}} \text { or } E=G \frac{M}{r^{2}}
$$

Where $\boldsymbol{k}$ is the proportionality coefficient of the charge of the gravitational cell of an ordinary body, where $\boldsymbol{k}=\mathbf{1}, \mathbf{0 3 8 9 3 1} \frac{\mathrm{m}^{3 / 2}}{\mathrm{C} \mathrm{s}}, \boldsymbol{k}^{2}=\mathbf{1 , 0 7 9} 378 \frac{\mathrm{m}^{3}}{\mathrm{C}^{2} \mathrm{~s}^{2}}$ $\boldsymbol{m}$ is the mass of the gravitational cell, where $\boldsymbol{m}=\mathbf{1 , 6 6 0 5 3 9} \cdot \mathbf{1 0}^{-\mathbf{2 7}} \mathrm{kg}$ $\boldsymbol{G}$ - gravitational constant, where $\boldsymbol{G}=\mathbf{6 , 6 7 4 3} \cdot \mathbf{1 0}^{-\mathbf{1 1}} \frac{\mathrm{m}^{3}}{\mathrm{~s}^{2} \mathrm{~kg}}$

The gravitational field around the gravitational cell at a distance of $\boldsymbol{r}=\mathbf{1} \mathrm{m}$ will be $\boldsymbol{g}=\boldsymbol{k}_{\mathbf{0}}^{2} \mathbf{4} \boldsymbol{q}_{0}{ }^{2}=1,108293 \cdot 10^{-37} \mathrm{~m} / \mathrm{s}^{2}$

Now let's explain the magnitude of $\boldsymbol{k}$ and $\boldsymbol{m}$. Let's start with $\boldsymbol{m}=$ $\mathbf{1 , 6 6 0 5 3 9} \cdot \mathbf{1 0}^{-\mathbf{2 7}} \mathrm{kg}$. To do this, imagine that two bodies of mass $\boldsymbol{M}$ and $\boldsymbol{M}_{\mathbf{1}}$ were formed from two black holes $\boldsymbol{M}^{\prime}$ and $\boldsymbol{M}_{\mathbf{1}}^{\prime}$. In this case, each gravitational cell of the black hole, due to the arrival of energy $\boldsymbol{E}$, increases its mass $\boldsymbol{m}_{\mathbf{0}}$ to mass $\boldsymbol{m}$ by the amount $\Delta \boldsymbol{m}$ (where $\Delta \boldsymbol{m}=\boldsymbol{E} / \mathrm{c}^{2}$ ). As a result, a plasma is formed from the superdense matter of a black hole, from which gaseous, liquid and solid substances can be formed. All four states of matter are neutral, that is, they have a total electric charge equal to zero. As a result of this circumstance, any substance can be represented as a huge set of gravitational cells. These cells consist of a proton and an electron with a total charge of $\mathbf{2} \boldsymbol{q}_{\mathbf{0}}$, as well as of neutrons, which are also a pair of a proton and an electron with a total charge of $\mathbf{2} \boldsymbol{q}_{\mathbf{0}}$. Thus, the mass of the 
gravitational cell $\boldsymbol{m}$ of any non-superdense substance (plasma, gas, liquid and solid) with high accuracy will be equal to $\mathbf{1} \mathbf{D a}$ (this is $1 / 12$ of the mass of an atom of the isotope of carbon-12) or $\mathbf{1 , 6 6 0 5 3 9} \cdot \mathbf{1 0}^{\mathbf{- 2 7}} \mathrm{kg}$. This implies that:

$$
k=\sqrt{\frac{G m}{4 q_{0}^{2}}}=\sqrt{\frac{6,6743 \cdot 10^{-11} m}{4 q_{0}^{2}}}=1,038931
$$

Thus, $\boldsymbol{k}>\boldsymbol{k}_{\mathbf{0}}$. This can be explained by the fact that, in contrast to the gravitational cell of a black hole, where everything is tightly compressed, in an ordinary cell there is an ultramicroscopic distance between elementary charges. As a result of this circumstance, more lines of force come out of an ordinary cell and go out, creating a $\mathbf{7 , 9 \%}$ stronger gravitational field in outer space. But due to the fact that the mass of an ordinary gravitational cell $\boldsymbol{m}$ is more than the mass of the gravitational cell of a black hole $\boldsymbol{m}_{\mathbf{0}}$ by $\mathbf{9 . 9 \%}$, then $1 \mathrm{~kg}$ of ordinary matter has a smaller number of gravitational cells than $1 \mathrm{~kg}$ of black hole matter. As a result of this circumstance, it turns out that $\boldsymbol{G}<\boldsymbol{G}_{\mathbf{0}}$, even though $\boldsymbol{k}>\boldsymbol{k}_{\mathbf{0}}$. For clarity, we will show this in a mathematical form:

$$
G=\frac{k^{2} 4 q_{0}^{2}}{m}=\frac{1,079378 \cdot 4 q_{0}^{2}}{1,660539 \cdot 10^{-27}}<G_{0}=\frac{k_{0}^{2} 4 q_{0}^{2}}{m_{0}}=\frac{4 q_{0}^{2}}{1,511593 \cdot 10^{-27}}
$$

Next, consider the gravitational interaction of a black hole with mass $\boldsymbol{M}_{\mathbf{0}}$ and an ordinary body with mass $\boldsymbol{M}$ at a distance $\boldsymbol{r}$ between them.

1. The gravitational field $\boldsymbol{E}$ around a black hole with mass $\boldsymbol{M}_{\mathbf{0}}$ will be:

$$
E=\frac{k_{0} k 4 q_{0}^{2}}{r^{2}} \frac{M_{0}}{m_{0}}=\frac{k_{0} k 4 q_{0}^{2}}{m_{0}} \frac{M_{0}}{r^{2}} \quad(1-13) \text { or } E=G_{1} \frac{M_{0}}{r^{2}}
$$


where $\boldsymbol{k}_{\mathbf{0}}=\mathbf{1}, \boldsymbol{k}=\mathbf{1}, \mathbf{0 3 8 9 3 1}, \boldsymbol{m}_{\mathbf{0}}=\mathbf{1 , 5 1 1 5 9 3} \cdot \mathbf{1 0}^{-27} \mathrm{~kg}$. Hence the gravitational constant is equal to:

$$
G_{1}=\frac{k_{0} k 4 q_{0}^{2}}{m_{0}}=\frac{1,038931 \cdot 4 q_{0}^{2}}{1,511593 \cdot 10^{-27}}=7,0572 \cdot 10^{-11} \frac{\mathrm{m}^{3}}{\mathrm{~s}^{2} \mathrm{~kg}}
$$

2. The gravitational field $\boldsymbol{E}$ around a black hole of mass $\boldsymbol{M}$ will be:

$$
E=\frac{k_{0} k 4 q_{0}^{2}}{r^{2}} \frac{M}{m}=\frac{k_{0} k 4 q_{0}^{2}}{m} \frac{M}{r^{2}} \quad(1-14) \text { or } E=G_{2} \frac{M}{r^{2}}
$$

where $\boldsymbol{k}_{\mathbf{0}}=\mathbf{1}, \boldsymbol{k}=\mathbf{1}, \mathbf{0 3 8 9 3 1}, \boldsymbol{m}=\mathbf{1}, \mathbf{6 6 0 5 3 9} \cdot \mathbf{1 0}^{-27} \mathrm{~kg}$. Hence the gravitational constant is equal to:

$$
G_{2}=\frac{k_{0} k 4 q_{0}^{2}}{m}=\frac{1,038931 \cdot 4 q_{0}^{2}}{1,660539 \cdot 10^{-27}}=6,4242 \cdot 10^{-11} \frac{\mathrm{m}^{3}}{\mathrm{~s}^{2} \mathrm{~kg}}
$$

Now we apply the gravity cells hypothesis to string theory. Consider first the gravitational interaction between black holes, and then move on to the gravitational interaction between ordinary masses.

The gravitational field between black holes $\boldsymbol{M}_{\mathbf{0 1}}$ and $\boldsymbol{M}_{\mathbf{0 2}}$ consists of many ultramicroscopic vibrating strings that connect the gravitational cells of black holes. These gravitational strings in their totality form a gravitational field in space. From the set of energies $\boldsymbol{e}$ of these strings, the total energy of the gravitational field between the bodies is added, that is, $\boldsymbol{E}=\sum \boldsymbol{e}$. The vibration energy of one gravitational string $\boldsymbol{e}$, which connects two gravitational cells, is a constant value and is numerically equal to $\boldsymbol{g}_{\mathbf{0}}(\mathbf{f} . \mathbf{1 - 3})$, that is:

$$
e=4 q_{0}^{2}=1,026789 \cdot 10^{-37} \mathrm{~J}
$$




\section{But a gravitational quantum, unlike an electromagnetic quantum}

(photon), does not move in space, but vibrates in the form of a long energy string connecting two gravitational cells of two different masses. Thus, we are dealing with a standing wave in the form of an oscillating string between two cells.

The energy source of the string is 4 elementary charges located in two gravitational cells. The cells vibrate with the same frequency together with the gravitational string, forming a single whole with the string. In contrast to the wellknown formula for the quantum of the electromagnetic field $\boldsymbol{e}=\boldsymbol{h} \boldsymbol{\gamma}$, the formula for the quantum of the gravitational field is as follows:

$$
e=h_{g} v R^{2} \text { or } e=h_{g} v \lambda^{2}(1-16)
$$

$\boldsymbol{e}$ is the energy of the quantum of the gravitational field, $\boldsymbol{e}=\mathbf{1 , 0 2 6 7 8 9} \cdot \mathbf{1 0}^{-\mathbf{3 7}} \mathrm{J}$. $\boldsymbol{h}_{\boldsymbol{g}}$ is constant for the case of gravitational interaction, where $\boldsymbol{h}_{\boldsymbol{g}}=\frac{\mathbf{1}}{\mathbf{4}} \boldsymbol{h}=$ $1,656518 \cdot 10^{-34} \mathrm{~kg} \cdot \mathrm{m}^{-1} \cdot \mathrm{s}^{-1}$

$\boldsymbol{v}$ is the vibration frequency of the gravitational string, $\mathrm{m} / \mathrm{s}$.

$\boldsymbol{R}$ is the distance between cells (bodies), $\mathrm{m}$.

$\boldsymbol{\lambda}$ - standing wave length between cells, $\mathrm{m}(\boldsymbol{\lambda}=\boldsymbol{R})$.

The constant $\boldsymbol{h}_{\boldsymbol{g}}$ and Planck's constant $\boldsymbol{h}$ are related, but at the same time different physical constants, because they reflect different physical processes. One of their main differences is that an electromagnetic quantum in the form of a photon moves in space, while a gravitational quantum does not move, but oscillates in the form of a standing wave between two cells in different bodies. 
Therefore, they have not only different dimensions: $\boldsymbol{h}_{\boldsymbol{g}} \mathrm{kg} \cdot \mathrm{m}^{-1} \cdot \mathrm{s}^{-1}, \boldsymbol{h} \mathrm{kg}$. $\mathrm{m}^{2} \cdot \mathrm{s}^{-1}$, but also different values: $\boldsymbol{h}_{\boldsymbol{g}}=\frac{\mathbf{1}}{\mathbf{4}} \boldsymbol{h}$. The fourfold difference can be easily explained. An electromagnetic quantum (photon) is formed as a result of oscillations of one charged particle (electron), and a gravitational quantum is the result of oscillations of 4 charges. In this case, all 4 charges are in a closed string system, which increases the frequency of oscillations of the gravitational quantum by 4 times in comparison with the electromagnetic quantum.

And now an important point. The proportion of the mass of the electron $\boldsymbol{m}_{\boldsymbol{e}}$ and the mass of the proton $\boldsymbol{m}_{\boldsymbol{p}}$ inside the gravitational cell is equal to the proportion of the energy of the gravitational quantum $\boldsymbol{e}$ to the constant $\boldsymbol{h}_{\boldsymbol{g}}$ :

$$
n=\frac{m_{e}}{m_{p}}=\frac{e}{h_{g}}
$$

Hence $n=\frac{e}{h_{g}}=\frac{4 e}{h}=\frac{4 \cdot 1,026789 \cdot 10^{-37}}{6,62607 \cdot 10^{-34}}=6,1984796 \cdot 10^{-4}$

(Note that the value of the proportion $\frac{4 e}{h}=6,1984796 \cdot \mathbf{1 0}^{-4}$ will be the same, both in the SI system and in the SGS system). Next, we get the formula for the mass of an electron:

$$
\boldsymbol{m}_{e}=\boldsymbol{m}_{p} \cdot \boldsymbol{n}
$$

Taking into account that $\boldsymbol{m}_{\boldsymbol{p}}=\boldsymbol{m}_{\mathbf{0}}-\boldsymbol{m}_{\boldsymbol{e}}$, we get: $\boldsymbol{m}_{\boldsymbol{e}}=\frac{\boldsymbol{m}_{\mathbf{0}} \boldsymbol{n}}{\mathbf{1 + n}}$. Let's substitute in this formula the values $\boldsymbol{m}_{\mathbf{0}}=\mathbf{1 , 5 1 1 5 9 3} \cdot \mathbf{1 0}^{-\mathbf{2 7}} \mathrm{kg}$ and $\boldsymbol{n}$ and we get the value of the electron mass in the gravitational cell:

$$
m_{e}=\frac{1,511593 \cdot 10^{-27} \cdot 6,1984796 \cdot 10^{-4}}{1+6,1984796 \cdot 10^{-4}}=9,363775 \cdot 10^{-31} \mathrm{~kg}
$$




\section{As you can see, the calculated electron mass almost completely}

coincided with the experimental mass of a free electron: $9,109389 \cdot 10^{-31}$

kg. The reason for the discrepancy by $\mathbf{2 , 7 \%}$ is the fact that in the gravitational cell of a black hole there is an insignificant increase in the mass of an electron in comparison with the mass of an electron in a free state. (It is important to note here that the electron and proton inside the gravitational cell of a black hole are not independent particles).

Further, taking into account that, $\boldsymbol{m}_{0}=\frac{\sqrt{8} \boldsymbol{q}_{0}}{c}, \boldsymbol{n}=\frac{\boldsymbol{e}}{\boldsymbol{h}_{g}}, \boldsymbol{h}_{g}=\frac{1}{4} \boldsymbol{h}, \boldsymbol{e}=$ $\mathbf{4} \boldsymbol{q}_{\mathbf{0}}{ }^{\mathbf{2}}$, we get the formula for the mass of an electron, expressed in terms of three fundamental constants:

$$
m_{e}=\frac{m_{0} n}{1+n}=\frac{32 \sqrt{2} q_{0}{ }^{3}}{c\left(h+16 q_{0}{ }^{2}\right)}
$$

In the case of gravitational interaction between ordinary bodies, the energy of the gravitational quantum between two cells is equal to $\boldsymbol{e}=\mathbf{1 , 1 0 8 2 9 3} \cdot \mathbf{1 0}^{-\mathbf{3 7}}$ $\mathrm{J}$ (1-11). As a result, according to the formula (1-18), we get:

$$
n=\frac{e}{h_{g}}=\frac{4 e}{h}=\frac{4 \cdot 1,108293 \cdot 10^{-37}}{6,62607 \cdot 10^{-34}}=6,69050 \cdot 10^{-4}
$$

Proportional equality remains the same: $\frac{\boldsymbol{m}_{e}}{\boldsymbol{m}_{\boldsymbol{p}}}=\frac{\boldsymbol{e}}{\boldsymbol{h}_{\boldsymbol{g}}}$. Hence, knowing the mass of the "ordinary" gravitational cell $\boldsymbol{m}=\mathbf{1}, \mathbf{6 6 0 5 3 9} \cdot \mathbf{1 0}^{-27} \mathrm{~kg}$, we determine the mass of the electron:

$$
m_{e}=\frac{m n}{1+n}=\frac{1,660539 \cdot 10^{-27} \cdot 6,69050 \cdot 10^{-4}}{1+6,69050 \cdot 10^{-4}}=11,102408 \cdot 10^{-31} \mathrm{~kg}
$$


As you can see, the mass of an electron in a "normal" gravitational cell is $18 \%$ greater than the mass of an electron in a superdense cell. This is due to an increase in the mass of the gravitational cell by $10 \%$ and the related structural changes within the cell. Thus, the mass of an electron in a superdense gravitational cell is closest to the mass of an electron in a free state.

Now, from formula 1-16, we obtain the formula for the vibrational velocity of a gravitational string:

$$
v=\frac{e}{h_{g} R^{2}} \quad(1-17)
$$

In this formula, $\frac{\boldsymbol{e}}{\boldsymbol{h}_{\boldsymbol{g}}}$ is a constant. Therefore, the changes in the vibration velocity of the string $\mathrm{v}$ depend only on the change in the distance $\boldsymbol{R}^{2}$ between the cells. (This can be compared to playing the guitar, where as you clamp the higher fret, the speed of the string decreases.)

The speed of any body and wave cannot exceed the speed of light $\boldsymbol{c}$.

Therefore, this also applies to the vibrational velocity of the gravitational quantum, $\boldsymbol{v} \leq \boldsymbol{c}$. It follows that the maximum vibrational speed $\boldsymbol{c}$ of the string should be equal to the speed of light: $\boldsymbol{v}_{\max }=2, \mathbf{9 9 7 9} \cdot \mathbf{1 0}^{\mathbf{8}} \mathrm{M} / \mathrm{c}$. This maximum speed $\boldsymbol{c}$ will correspond to the minimum length of a standing gravitational wave $\boldsymbol{\lambda}_{\min }$ or the minimum distance $\boldsymbol{d}_{\text {min }}$ between cells, when gravitational forces are still acting. Further, from formula 1-17, we obtain the formula $\boldsymbol{d}_{\min }$ for the case of gravitational interaction between two ordinary (not superdense) bodies:

$$
d_{\text {min }}=\sqrt{\frac{e}{h_{g} c}} \sqrt{\frac{1,108293 \cdot 10^{-37}}{1,656518 \cdot 10^{-34 \cdot 2,9979 \cdot 10^{8}}}}=1,4937 \cdot 10^{-6} \mathrm{~m}
$$


Thus, the boundary of the transition $\left(d_{\min }=1,4937\right.$ microns $)$ of gravitational forces into much stronger electromagnetic forces has now been determined. A direct confirmation of this is the recorded scientific fact obtained in the experimental study of the Casimir effect (force). During these experiments, it was recorded that when two mirror-polished metal plates approach each other, $\underline{\text { it is }}$ at a distance of several micrometers between them that a strong attraction arises (at a distance greater than a few micrometers, the Casimir force is absent). Thus, the boundary of the action of gravitational forces calculated by the formula coincided with absolute accuracy with the boundary of the appearance of the Casimir effect, which is of electromagnetic origin and therefore belongs to electromagnetic forces.

Further we will get one more relationship of physical quantities. Based on formulas (1-5) and (1-15), the gravitational constant in the black hole region $\boldsymbol{G}_{\mathbf{0}}=$ $\frac{\boldsymbol{e}}{\boldsymbol{m}_{\mathbf{0}}}$. Taking into account that $\boldsymbol{e}=\boldsymbol{h}_{\boldsymbol{g}} \cdot \boldsymbol{n}, \boldsymbol{h}_{\boldsymbol{g}}=\frac{1}{\mathbf{4}} \boldsymbol{h}, \boldsymbol{n}=\frac{\boldsymbol{m}_{\boldsymbol{e}}}{\boldsymbol{m}_{\mathbf{0}}-\boldsymbol{m}_{\boldsymbol{e}}}$ we obtain the formula for the gravitational constant in the black hole region:

$$
G_{0}=\frac{e}{m_{0}}=\frac{h m_{e}}{4 m_{0}\left(m_{0}-m_{e}\right)}
$$

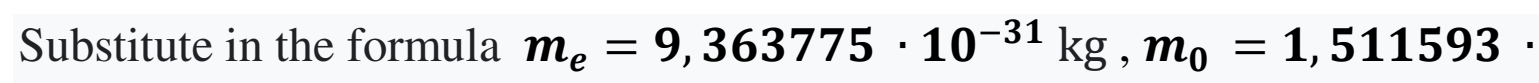
$\mathbf{1 0}^{-27} \mathrm{~kg}$, and get the value $\boldsymbol{G}_{\mathbf{0}}=\mathbf{6}, \mathbf{7 9 2 7} \cdot \mathbf{1 0}^{-\mathbf{1 1}}$.

The value of the "usual" gravitational constant $\boldsymbol{G}$ is determined by a similar formula. Taking into account that $\boldsymbol{m}=\mathbf{1}, \mathbf{6 6 0 5 3 9} \cdot \mathbf{1 0}^{-\mathbf{2 7}} \mathrm{kg}, \boldsymbol{m}_{\boldsymbol{e}}=$ $11,102408 \cdot 1^{-31} \mathrm{~kg}$, we get: 


$$
G=\frac{e}{m}=\frac{h m_{e}}{4 m\left(m-m_{e}\right)}=6,6743 \cdot 10^{-11}
$$

The formula for the speed of light in the gravitational field of black holes is of scientific interest. The formula is derived from: $\boldsymbol{G}_{\mathbf{0}}=\frac{\boldsymbol{e}}{\boldsymbol{m}_{\mathbf{0}}}$ and $\boldsymbol{G}_{\mathbf{0}}=\frac{\boldsymbol{m}_{0} \boldsymbol{c}^{2}}{2}(1-9)$ :

$$
c=\sqrt{\frac{2 e}{m_{0}{ }^{2}}}=\sqrt{\frac{2 \cdot 1,026789 \cdot 10^{-37}}{\left(1,511593 \cdot 10^{-27}\right)^{2}}}=2,9979 \cdot 10^{-8} \mathrm{~m} / \mathrm{s}
$$

(For better perception in the higher formulas, conversion factors for units of measurement are not specially set, which are equal to 1).

\section{Results and discussion.}

In this study, gravitational constants were determined for different cases of gravitational interaction.

1. $\boldsymbol{G}_{\mathbf{0}}=\mathbf{6}, \mathbf{7 9 2 7} \cdot \mathbf{1 0}^{-\mathbf{1 1}}$ (for the case of interaction of two black holes).

2. $\boldsymbol{G}=\mathbf{6}, \mathbf{6 7 4 3} \cdot \mathbf{1 0}^{-\mathbf{1 1}}$ (for the case of interaction of two ordinary bodies).

3. $\boldsymbol{G}_{1}=\mathbf{7}, \mathbf{0 5 7 2} \cdot \mathbf{1 0}^{-11}$ and $\boldsymbol{G}_{\mathbf{2}}=\mathbf{6}, \mathbf{4 2 4 2} \cdot \mathbf{1 0}^{-11}$ (for the case of interaction between a black hole and an ordinary body).

It is necessary to explain the third case of gravitational interaction, where there are two values of the gravitational constants $\boldsymbol{G}_{\mathbf{1}}$ and $\boldsymbol{G}_{\mathbf{2}}$. The presence of two gravitational constants can raise the question: "Isn't there a violation of Newton's third law?" There is no violation here, because throughout the study, mass was only a measure of the amount of matter, and nowhere did it act as a gravitational and inertial mass. This is clearly seen from formulas (1-13) and (114), in which the masses $\boldsymbol{M}_{\mathbf{0}}$ and $\boldsymbol{M}$ show only the number of gravitational cells in 
the body: $\boldsymbol{n}=\frac{\boldsymbol{M}_{\mathbf{0}}}{\boldsymbol{m}_{\mathbf{0}}}$ and $\boldsymbol{n}=\frac{\boldsymbol{M}}{\boldsymbol{m}}$. Therefore, Newton's third law $\left(\boldsymbol{F}_{\mathbf{1}}=-\boldsymbol{F}_{\mathbf{2}}\right)$, where there is inertial mass $\left(\boldsymbol{m}_{\mathbf{1}} \boldsymbol{a}_{\mathbf{1}}=-\boldsymbol{m}_{\mathbf{2}} \boldsymbol{a}_{\mathbf{2}}\right)$, cannot be applied here.

When discussing the research results, it is necessary to pay attention to the following point. The force of interaction between two elementary charges $\boldsymbol{q}_{\mathbf{0}}$ inside the gravitational cell is relatively large and is determined by the formula (11), where the proportionality coefficient is $\boldsymbol{k}=\mathbf{9} \cdot \mathbf{1 0}^{\mathbf{9}}$. But outside the gravitational cell, the proportionality coefficient $\boldsymbol{k}$ decreases sharply and for superdense gravitational cells it becomes equal to 1 , for ordinary gravitational cells

$-\mathbf{1 , 0 3 8 9 3 1}$. This circumstance $9 \cdot \mathbf{1 0}^{9}$ times weakens the electric field outside the gravitational cell, turning it into a gravitational field.

Another result of this study is the determination of the mass of the gravitational cell of the black hole $\boldsymbol{m}_{\mathbf{0}}=\mathbf{1}, \mathbf{5 1 1 5 9 3} \cdot \mathbf{1 0}^{-\mathbf{2 7}} \mathrm{kg}$. Gravitational cells with a mass less than $\boldsymbol{m}_{\mathbf{0}}$ do not exist.

The introduction to physics of the concept of a gravitational quantum in the form of an oscillating energy string is one of the main results of this research. The formula for the energy of a gravitational quantum was obtained. Based on this formula, a formula for the mass of an electron was obtained on the basis of three fundamental constants. Further, using this formula, the electron mass was calculated, which coincided with the known mass of a free electron. This should be attributed to important scientific results.

Also, according to the formula for the energy of a gravitational quantum, the exact boundary of the transition of gravitational forces into electromagnetic forces 
was determined, which is the distance of $\boldsymbol{d}_{\min }=\mathbf{1}, \mathbf{4 9 3 7}$ microns between bodies. The minimum distance $\boldsymbol{d}_{\min }$ calculated by the formula coincided with absolute accuracy with the known experimental data obtained in the study of the Casimir effect (force). This circumstance confirms the correctness of the new formula and the result obtained. It should be noted that $\boldsymbol{d}_{\min }$ is simultaneously the minimum

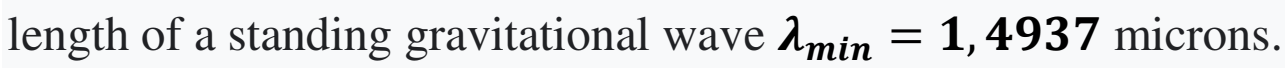

The scientific result is also obtaining a formula for the gravitational constant based on Planck's constant, the mass of the electron and proton.

\section{Conclusions.}

The main result of this research is the embedding of the hypothesis of gravitational cells in string theory and the subsequent application of the augmented theory to one of the fundamental interactions - gravitational interaction. This avoided the so-called landscape problem in string theory. As a result of the application of the hypothesis of gravitational cells and the concept of string theory, a formula for the gravitational constant in the region of black holes was obtained and its value was determined: $G_{0}=\sqrt{2} q_{0} c=6,7927$. $\mathbf{1 0}^{\mathbf{- 1 1}}$. Also received justification and confirmation of the value of the "ordinary" gravitational constant $G=6,6743 \cdot 10^{-11}$.

In this study, the mass of the gravitational cell of a black hole was determined, where $\boldsymbol{m}_{\mathbf{0}}=\mathbf{1}, \mathbf{5 1 1 5 9 3} \cdot \mathbf{1 0}^{-\mathbf{2 7}} \mathrm{kg}$. (The mass of a gravitational cell 
can be attributed to new physical constants, because gravitational cells with a mass less than $\boldsymbol{m}_{\mathbf{0}}$ do not exist).

\section{Through augmented string theory, it was possible to apply Planck's} formula to the gravitational interaction of bodies. As a result, the formula for the quantum of the gravitational field and the formula for the vibrational velocity of gravitational strings were obtained. (This result assumes the existence of a similar electric field quantum in the form of an oscillating string between two elementary charges, where $\left.\boldsymbol{e}=\mathbf{9} \cdot \mathbf{1 0}^{9} \boldsymbol{q}^{2}=\mathbf{2 , 3 1 0 2 7 5} \cdot \mathbf{1 0}^{-28} \mathrm{~J}\right)$. Further, on this theoretical basis, a formula was obtained to determine the mass of an electron. The electron mass calculated by this formula turned out to be equal to the experimental electron mass.

In this scientific work, the boundary of the transition of gravitational forces $d_{\min }=1,4937$ microns into electromagnetic forces was precisely determined for the first time. An experimental confirmation of this is the fact that, when mirrorpolished metal plates approach each other, the Casimir effect appears at a distance of several micrometers. With a larger distance between the plates, the Casimir force is absent. Thus, the well-known experiment has confirmed that the distance $\boldsymbol{d}_{\min }=\mathbf{1}, 4937$ microns between bodies is the boundary of the transition of gravitational forces into stronger electromagnetic forces. It should be noted that $\boldsymbol{d}_{\boldsymbol{m i n}}$ is simultaneously the minimum length of the standing gravitational wave $\lambda_{\min }=1,4937$ microns. 
Thus, based on the unification of the hypothesis of gravitational cells and string theory, in addition to theoretical achievements, three specific practical results were obtained. These are the value of the gravitational constant calculated by the new formulas, the value of the electron mass and the value of the minimum distance for the beginning of the gravitational interaction. The calculated values coincided with the known experimental data. Threefold coincidence of the final results on the same theoretical basis absolutely excludes the element of chance.

Also, based on the results obtained, it can be concluded that in a free state, the proton and the electron do not have gravitational mass. Only when these particles come close together, a gravitational field is formed around them.

A detailed interpretation of the results obtained in this study will be given in the next article. Now at this stage, it is important to consolidate the results obtained. 


\section{Declarations}

1. Availability of data and materials.

All data obtained and analyzed in the course of this study is included in this article.

2. Competing interests. Not applicable (there are no competing interests).

3. Funding. Not applicable.

4. Authors' contributions. Not applicable.

5. Acknowledgements. Not applicable. 